\title{
Manufacturing Interfaces
}

\author{
F. J. A. M. van Houten, University of Twente
}

\begin{abstract}
The paper identifies the changing needs and requirements with respect to the interfacing of manufacturing functions. It considers the manufacturing system, its components and their relationships from the technological and logistic point of view, against the background of concurrent engineering. Design- and manufacturing features are considered to become the basic elements for both internal and external communication between manufacturing functions. The increasing level of automation on the shop floor requires a much more formal communication at a high level of detail. Together with the increasing need for flexibility and the resulting decrease of batch sizes, this demands a much closer integration of production planning process planning and shop floor control. Improvement of communication in combination with the use of feed-back and shop floor control. Improvement of communication in combination with the use of feed-back data from the shop floor can substantially increase the iffectiveness of the planning and consequently reduce the time pressure on the manufacturing system and its operators. Planning and control of auxiliary tasks and resources like tool and fixture preparation, machine set-up, material preparation, etc. increases due date reliability and quality and lowers production cost.
\end{abstract}

Koywords: Interfaces, CAD/CAM, automation, optimization, production control, CIM

Acknowledgement

Valuable contributions to this keynote paper were received from B.J. Davies, J.P Kruth, M.E. Merchant, A. Novak, G. Sohlenius and C.A. van Luttervelt.

\section{Introduction}

This paper reflects the scope of interest of the STC " $\mathrm{O}$ " working group on Manufacturing Interfaces. This working group, which was originally called "Communication Interfaces" has been established in 1987 with the objective to follow the developments in standardization of communication media and protocols for the manufacturing environment, such as the Manufacturing Automation Protocol (MAP). One of the first activities of the group was the preparation of a survey on the interest of CIRP members in topics like:

- Industrial local and wide area networks.

- Networking standards.

- Communication protocols (Manufacturing Message Specification, etc.)

- High level languages for communication within FMS

- Operator interfaces for FMS

- CAD interfacing (PDES, STEP, IGES, SET, VDA-xS, etc.)

- NC interfacing standards

- User interfaces for CAD/CAM

- CAD/CAM interfacing

Response to the survey was received from 24 members and showed that interest existed in all of these topics. However, most of the actual research work mentioned by the respondents was carried out in the last category.

The scope of interest of the group gradually extended to more general issues concerning the interfacing of manufacturing functions and the emphasis of the discussions was no longer put on the specific implementation details of the interfaces (the "HOW") but more on the functional requirements of information transport between manufacturing functions (the "WHAT".). This was illustrated by the groups name change from "Communication Interfaces" to "Manufacturing Interfaces" in August 1990. (According to CIRP terminology, "Manufacturing" should be understood here as including all activities from product design to realization. However, in the rest of this paper, it has been impossible to be absolutely consequent and to avoid the common use of the term which indicates the part realization function only, like in "Design for Manufacturing")

This keynote paper represents the reflection of several meetings of the working group in which the changing requirements of the manufacturing environment and their consequences with respect to the interfacing between manufacturing functions have been discussed. These discussions resulted in the set of diagrams which are explained in this paper (fig. 1-3 and 7-12).

\section{Changing requirements for the manufacturing industry}

\subsection{The changing market}

The fierce competition on the international market forces most companies to change their production strategy. New products must be developed quicker, manufacturing methods must be renewed and the manufacturing organization must be adapted to the demands for shorter delivery times, higher product quality and higher flexibility (more product variants in smaller batch sizes) at acceptable prices.

Quality is rapidly becoming a very important market factor. Quality certification (ISO 9000-9003) requires a thoroughly documented manufacturing process. This requires formalisation and control of communication processes within the organization. Quality assurance should be implemented as part of the formal information structure. be held responsible for the support of the total product life cycle, including disassembly and recycling of materials [1].

\subsection{The need for a tighter integration of manufacturing functions.}

In the traditional sequential approach to product creation and realization, the first phase is carried out by the marketing function, which translates customer requirements in functional product requirements. Subsequently, the design function is mainly focused on the translation of functional requirements into a product definition which can be recorded in terms of structure, shape and material specifications. A design is usually completely finished before any other activity is started. Because of that, the consequences of the design decisions for the subsequent processes first become clear during process planning (when the product structure has to be converted into a process structure). The manufacturing production processes, (which, according to CIRP terminology, include assembly and coating) but also the disassembly and recycling processes put constraints on the range of product shapes, accuracies and material properties which can be realized. Designers which do not consider these constraints sufficiently, produce designs which may be good from a functional point of view but which cannot be produced economically and are not acceptable with respect to maintenance and recycling. Terms like "Design for Manufacturing", "Design for Assembly" and "Design for Disassembly" reflect the awareness of this problem in the design community. They indicate the need for better procedures which assist designers in converting functional product requirements into shape/material combinations which can be produced, assembled and recycled in an economic way.

\subsection{The need for concurrency}

A substantial reduction in time-to-market can be achieved by early involvement of representatives of all production functions, like marketing, sales, purchasing, design, engineering, planning, parts production, assembly, recycling etc. Ample communication with subcontractors during the early stages of product development also contributes to the goal, in particular if any design and engineering tasks are subcontracted (co-makership). Concurrent engineering (also called simultaneous engineering [2]) is a concept which abandons the sequential execution of design and manufacturing production activities. According to this concept, the engineering and planning of the parts manufacturing, assembly, maintenance, disassembly and recycling processes should be carried out concurrently with the development of the product. Running these activities in parallel can speed up the development of new products substantially and will improve their overall quality. However, this is easier said than done. True concurrent engineering is not possible yet with the present generation of engineering tools. A seamless continuous connection between all design, engineering and manufacturing production functions is an Utopia, unless all activities are performed by one and the same person (like the blacksmith in the past).

\subsection{Consequences for the communication between manufacturing functions}

The wish to run activities in parallel puts a heavy burden on the communication infrastructure of the company. The concept of the diversification of labour, as promoted by F. W. Taylor, has increased the efficiency of the individual manufacturing functions substantially because of the resulting specialization. However, in the mean time it has resulted in complex company structures with a multitude of departments, each of them having their own responsibilities. A typical effect of a strict departmental organisation structure is that local goals tend to prevail upon the overall goals. In this type of environment, many discussions about how to reach the overall goals of the company develop into to quarrels about who is responsible for which sub-function and subsequently come to a deadlock. This lack of cooperation is mainly caused by the fact that specialists do have problems to appreciate the relativity of their own decisions when they are confronted with a larger frame of reference. Cooperation is stimulated if 
information, which is generated by a given function, is supplied as soon as possible to other functions.

In every organization many formal procedures for aking decisions and treating information do exist. Although these procedures are usually based on the sequential execution of the different functions, the amount of data to be handled is already large. The intention to run functions in parallel will increase the need for information flow substantially. In order to avoid information congestion, adequate information filtering, storage and retrieval systems are required. High performance communication systems are needed. But before all, the interfaces between the manufacturing functions as required by concurrent engineering have to be defined and implemented.

\subsection{Consequences for the interfaces between manufacturing functions}

The major problem of manufacturing interfaces is that they were never really designed as an integral part of a manufacturing system. In the past, most manufacturing systems grew gradually by addition of extra personnel and equipment to existing facilities. Only in some cases, plants were designed for specific products. Communication was considered to be the task of the people within the system. They had to read instructions, watch indicators, push buttons and fill in forms. Automation was implemented island-wise and electronic means of communication were added to the systems as needs arose, if technically feasible. Many local automation solutions have been developed and installed during the last decades. Because these systems have been specified and developed by specialists, they are tailored for the specific functions, have proprietary data representations and usually have poor facilities for information exchange with other functions. In order to start the implementation of concurrent engineering systems, the interfacing between the available system components has to be improved and new developments in the field must be compatible with the requirements of concurrent engineering.

\subsection{User interfaces}

The development of system-to-system interfaces alone is not sufficient. Human communication will continue to have a tremendous influence on the efficiency of manufacturing systems. The development and improvement of user interfaces which provide access to the manufacturing equipment controllers, to the supporting programs for the higher level manufacturing functions and to systems for human-to-human communication, is equally important. Concurrent engineering in combination with flexible manufacturing requires a very communicative organization, capable to deal with very detailed information which is frequently exchanged between the various functions. It is important that this information is presented in a very transparent way to all the people involved in order to enable them to anticipate on upcoming problems. The quality of the user interfaces to the complicated networks of design and manufacturing functions is crucial for the acceptance by the people who have to work with them. Instead of exploiting the Taylorian principle of diversification of tasks and the related limited responsibility for the delivery of the product, a high level of involvement in related functions should be stimulated by the application of up to date communication technology. This requires that the users of automated systems must have access to more than just the bare information they need for doing their own job. For instance, if an operator on the shop floor gets information about a set of jobs which have to be performed after the current one, he can anticipate on lacking materials, tools or data. He can contact the production and process planning people about sequences of jobs which are in his opinion illogical etc. Process planners can contact designers about manufacturability problems and vice versa. Production planning can retrieve information about the actual situation on the shop floor. All information has of course to be protected against unauthorized access but in principle most of the data should be accessible for most functions.

\subsection{Training needs}

No technical system can function properly without well trained operators and maintenance people. This implies that operators have to be taught how to use the equipment and its communication facilities efficiently and engineers have to be trained to design, install and maintain the systems and their interfaces.

\section{The influence of production system typology on the requirements for concurrency and flexibility}

Automation on the shop floor has increased the efficiency and productivity of most companies, but usually at the cost of a reduced flexibility.

\subsection{High volume production}

In high volume production, some flexibility has been regained by installing equipment which can execute different programs on call. The manufacturing handling and transport equipment is fully automated and it is coordinated cluster wise by supervisory control systems. It is possible to produce (a limited set of variants on customer order. The overall flexibility of the systems is mainly determined by the reliability of the individual processes as well as by the supervisory control system itself. The control is simplified by the fixed availability of information in the equipment controllers and the majority of the information flow consists of only trigger messages and status information. In high volume production, the production facility with all its manufacturing resources is usually planned on beforehand, together with the design and engineering of the product(s). In terms of concurrent engineering, the emphasis is put on the simultaneous development of the product(s), the processes, the equipment and the layout of the factory.

This paper does not explicitly deal with non-order based production of larger batches, where simultaneous design of products, production processes and production facilities is required, but that theme is discussed in the keynote paper "Concurrent Engineering" by G. Sohlenius [2].

\subsection{Small batch production}

In small batch production, orders have to compete for a more or less fixed set of manufacturing resources. Technological and logistic planning are continuous on-line activities and the products have to be manufactured within the constraints of the available resources. This requires a continuous tight integration of design, process planning, production planning and shop floor control. The product mix has a large variety and every order has to be evaluated with respect to the suitability and capacity of the available manufacturing resources. In this type of manufacturing environment, the emphasis is put on the decrease of throughput times and the decrease of the amount of work in progress in combination with an acceptable average utilisation of the equipment and a proper balance of the workload over the resources.

Work-in-progress can be reduced if the manufacturing activities are planned in a very detailed and accurate way, if the execution of jobs is performed exactly according to plan and if waiting times, which cause under-utilization of manufacturing resources, are avoided. Production cost can be reduced by an increase of the average utilisation of manufacturing resources, the improvement of process reliability and process performance and by the standardisation of tools and manufacturing methods.

Master planning decides on the basis of the product structure and the bill of materials in which planning period specific parts and sub-assemblies have to be produced. It has to juggle with order priorities because of the limited capacity of the resources and needs information from process planning about estimated cycle times and the global capacity of the resources, in order to be able to determine reliable internal due dates. The master planning has to be refined by local planning functions, like capacity planning and scheduling. Feasible combinations of production orders and specific resources have to be determined by process planning. Capacity planning has to produce workplans which should be based on a realistic capacity utilization and allow delivery of all jobs according to the internal due dates. The workplans should be sufficiently tolerant for disturbances like rush orders and break downs. The relatively high number of jobs with a low work content makes production planning very complicated. The information flow consist of a complex mix of workplans, schedules, job control information, NC-programs, set-up information, operator instructions, tool offset data, status information, trigger messages, etc.

This paper focuses particularly on small batch order-based production with (more or less) fixed sets of resources. Although, in this kind of manufacturing environment, the manufacturing resources and processes are usually not designed simultaneously with the product, it is very important that generic manufacturability constraints are considered during the product design phase. Standardisation of manufacturable design elements and a tight integration of design and process planning contributes to the improvement of the overall efficiency of the manufacturing system.

\section{$4 \mathrm{~A}$ reference diagram for the interfacing of manufacturing functions in} small batch production

Figure 1 shows a reference diagram of the manufacturing functions with their interfaces, which will be discussed in the paper. The diagram has to be interpreted as the representation of a highly communicative manufacturing environment which can be realized with present day technology. However, it does not necessarily represent a specific implementation. The diagram is based on an elaboration of the NIST factory model and a shop floor control model which has been developed in ESPRIT project 809 [3]. A series of figures which show details of the first diagram, will be used in the following chapters to indicate important manufacturing interfaces.

In the diagram, the manufacturing system is split up in different levels, each comprising a number of related functions. The factory level includes purchasing, sales/order intake, design \& engineering and master planning. On the system level the process planning, capacity planining and quality assurance functions are situated. The cell level comprises the scheduling, dispatching and monitoring/diagnostics functions as well as the resource databases and related information support functions. The workstation level contains the so called workstation controller functions, auxiliary station controller functions and shop floor communication functions. Examples of auxiliary stations are the tool room and the material store. On the equipment level the machine 


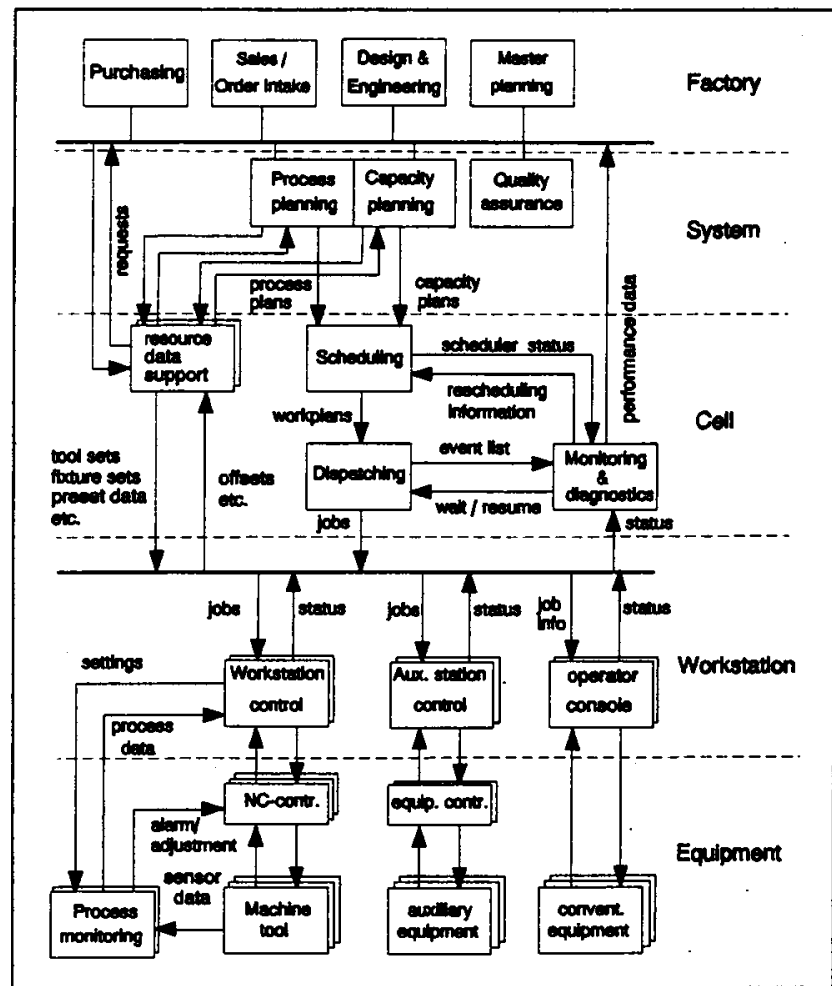

Fig. 1 The reference diagram for the identification of manufacturing functions

tools (with their controllers and process monitoring equipment) and the actual presetting, storage, transport, handling and measurement systems are situated.

The diagram relates to a hierarchical control concept. In principle higher levels control lower levels. However, this does not imply that al communication between the different functions has to follow the "vertical structure" of the hierarchy. "Horizontal communication" between functions on the same level is equally essential.

The diagram shows the design functions on top and the machining functions at the bottom. Although products have to be designed before they can be manufactured, the diagram does not implicitly include a timely ordering of the functions from top to bottom. This means that the functions on the different levels can (partially) be executed in parallel and concurrency is not hampered by the control hierarchy.

The boxes in the diagram represent collections of sub-functions which are classified under names referring to a decomposition of the manufacturing system according to the traditional terminology. However, some recent implementations of modular systems allow sub-functions to be integrated within function modules belonging to a different category (such as process planning sub-functions as part of a design system or capacity planning subfunctions as part of a process planning system etc.)

\section{Coatrol and feed back in automated manufacturing systems}

\subsection{Flexible manufacturing systems and $\mathrm{CIM}$}

A major step towards integration of manufacturing processes was the concept of FMS (Flexible Manufacturing Systems): Conglomerates of tightly coupled NC machine tools with automated material handling, tool handling and transport devices. The controllers of these systems are linked with each other and are connected with a supervisory control system. Local area networks created the possibility to connect CAD/CAM functions in the office with the DNC (Direct or Distributed Numerical Control) networks on the shop floor. The interfacing of CAD/CAM/DNC with logistic information systems is called CIM (Computer Integrated Manufacturing). In the early eighties, the combination of the concepts CIM and FMS have raised high expectations about the feasibility of the fully automated factory. Unfortunately, FMS cannot be used effectively in true small batch manufacturing due to the complex technological and logistic planning problems and the limited predictability of the processes.

\subsection{Flexible manufacturing cells}

Most experts in the field of small batch manufacturing agree that aiming at a slightly lower degree of automation and integration is much more realistic and economic. The typical machine shop of today consists of a number of CNC machine tools and auxiliary equipment, usually grouped in manufacturing cells, according to the classification of the product mix. Set- up, transport and manipulation functions, which have not been automated, are carried out by human operators. They supervise the technological and logistic processes on the shop floor. As mentioned before, it is very important that the operators are being supplied with sufficient information, not only about the current manufacnuring job but also about the next ones. This enables them to anticipate on possible problems which have not been foreseen by the preparatory functions. Early detection of discrepancies between the planned and actual behaviour of the systems can avoid propagation of disturbances

Production planning and process planning are usually carried out in a central department. Tool and material management are often also centralized. The main goal of the planning department is to keep the amount of work-inprogress low and the utilisation of the equipment high. This is pursued by a rather detailed planning of activities, resources and technological process conditions. However, in most situations, the discrepancies between the planning and the actual situation on the shop floor are still quite large.

\subsection{Improvement of control by the use of feed-back information}

The material and information flow on the shop floor must be monitored very accurately in order to detect disturbances which may affect dependent manufacturing operations. The propagation of disturbances may delay many operations and invalidate the production plan. In that case, urgent production orders have to be re-allocated to other resources and new process plans have to be made. This requires the capability of on-line process planning and a very flexible way of production planning. Feed-back of progress and performance information is of vital importance for the responsiveness of the manufacturing system to disturbances like shortages, break-downs and rush orders [4]. Performance data should be analyzed and the results should be used for the adjustment of the models which predict the behaviour of the manufacturing system. Process planning should include the optimization of process conditions according to various objective functions. The resulting process behaviour should be monitored and fed back to the relevant system levels.

Real-time control requires short response times in the lower level feed back loops, while data reduction has to avoid congestion in the higher level loops. As a consequence, the communication requirements of the control loops vary substantially. Concepts of multi level adaptive control systems bave been described in [5] and [6]. Figure 2 shows the hierarchical control levels and feed back loops which are required in automated small batch part manufacturing systems. The existence of process, flow, performance and cost monitoring is the backbone of CIM technology.

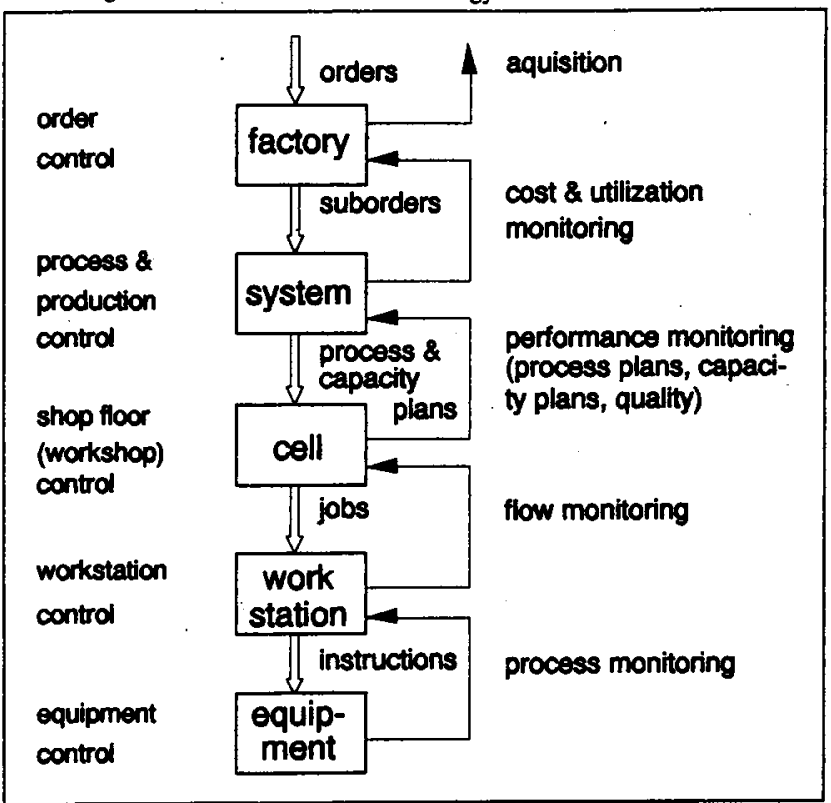

Fig. 2 Hierarchical control levels and loops in small batch part manufacturing

\section{Limitations of existing automation solutions}

\subsection{Design}

Product development time and cost can be reduced substantially by the re-use of engineering and manufacturing knowledge during the design stage. However, the $\mathrm{CAD} / \mathrm{CAM}$ systems which are presently used in industry do not support this very well. The systems merely offer storage and retrieval facilities for product models on the low level of (sometimes poorly structured) geometric entities.

The present generation of CAD/CAM systems offers hardly any design and process planning functionality. However, most systems allow customization by means of (dedicated) programming languages. Application programs made 
by the user can improve the productivity of CAD systems quite substantially However, in practice, most companies work with (a very limited sub-set of the standard functionality of their CAD systems. This is mainly due to the fact that the CAD operators have difficulties with the large and sometimes illogically organised command set, which represents too much of the CAD system's intermal structure and too little of the application domain.

The exchange of product model data between different $\mathrm{CAD}$ and CAE systems is rather tedious because of the differences in the internal geometry representations and the ambiguous definition of the exchange standards. CAD proliferates rapidly but many companies still use it only to produce the equivalent of traditional drawings.

\subsection{Master production planning}

The traditional production planning methods like Materials Requirement Planning (MRP) and Manufacturing Resource Planning (MRPI) are "assembly" driven and manufacturing operations are planned backwards. MRP plans against unlimited resource capacity, while MRPII takes only the capacity of the primary capacity resources (machine tools) into account. The smallest unit of work is in the order of one operation per week, which make MRP unsuitable for the planning of small batches. Production planning for small batches of products which require many operations has to be performed on a much smaller timescale. Strong links are needed with capacity planning, process and operations planning and shop floor control.

\subsection{Process planning}

In the past, process planning automation has mainly been based on storage retrieval and editing of documents on which the process steps are listed Variant systems, based on part classification and group technology are used to cluster small batches to larger ones and to reduce the number of different routings through the workshop. These systems are mainly useful for the planning of manufacturing operations on conventional machines. NC machining requires a much higher level of detail in description of the individual operations, the tool path, tool geometry and offsets, the fixtures and set-ups, the process conditions etc. Only generative process and operations planning systems are suitable for this purpose. Supercapes ${ }^{\star}$ and MetCapp are examples of (semi) generative CAPP systems, presently used in industry. The degree of automation is still rather low and the interfacing to both the design and CAM functions are limited.

\subsection{Numerical control}

The limited programmability of the early Numerical Controllers in combination with the average complexity of the parts which had to be produced with them, forced the users to have the NC programming carried out in a central department, using higher level NC programming languages like APT. The decisions related to the execution of manufacturing processes were not longer taken by the machine tool operator but became the full responsibility of the process and operations planner. For the planning department, this implicated the need for a much more detailed knowledge about the actual sinuation at the shop floor and a much more formal organization of the workshop. Because of the high cost of the machines and the complexity of the programming, NC machines were mainly used in large companies. These companies could also afford to use the early generation CAD systems for the creation of NC tool path geometry.

For the smaller companies, the introduction of CNC increased the possibility to use automated machine tools because no drastic changes in the organization of the company were required. On the basis of traditional (or CAD) drawings, the machines can be interactively programmed on the shop floor by their operators, who also carry out all the peripheral tasks like setting-up, tool management, etc. As such, these self-supporting CNC units offer a very high local flexibility. However, the fixed combination of operators and machines makes the workshops as a whole rather vulnerable and inflexible with respect to reallocation of production orders and/or operators. A remedy can be found in shop floor oriented NC programming systems which can be used by the machine tool operators. Most of these systems can accept digital product data from CAD systems, which eliminates the need for complete re specification of product geometry and eases porting of NC programs to other machines. However, the interfacing to other technical and logistical information systems is virtually non existent.

For cutting operations, the format of NC part programs is standardized but there are many ambiguities. NC programs for the same product on different machines can show considerable differences. This still causes problems in interfacing CAM and NC programming systems to NC machines. The required postprocessors have to be bought from the CAM vendor or have to be made by the user with the help of a postprocessor generator. Postprocessors are a nuisance for many companies. A collection of good working postprocessors usually forms a threshold for making a change from one CAM system to another.

For most other operations like grinding, EDM, laser machining, sheet metal fabrication, etc. the programming chaos is complete. Virtually each solution is a specific one. This is mainly because of the fact that a significant part of the process technology is embedded in the machine and cannot be programmed in a "standard" way (for EDM in the generator, for laser machining in the beam, the optics, the gas flow etc.). However, ISO is actively developing new standards and reworking existing ones for CAM and NC programming purposes. (ISO 3592 defines $\mathrm{CL}$-data, the unidirectional interface between CAM systems and postprocessors, ISO 4343 defines the postprocessor vocabulary and ISO 4342 describes the APT language). The new standards will allow to support more advanced types of NC controllers and will be extended with new type of records for supporting non-traditional machine tools as well, like EDM and LBM machines [7]

The physical interfacing to NC controllers is still quite primitive, compared to the standards of general purpose computing. The MAP effort to standardize the communication between shop floor equipment and higher level systems was initiated by General Motors in the early eighties. Unfortunately the progress was very slow and the proliferation of the Manufacturing Automation Protocol is not very much stimulated by the NC controller and computer vendors. Presently, most connections to NC controllers are still based on serial communication (RS232). The expressiveness of the NC language is very poor. Tool paths for free-form surfaces have to be specified in huge sets of coordinates. For these large NC programs which usually have to be loaded in several blocks (because of memory limitations of the controller) the limited data transmission speed often is an annoying restriction to the feed rate during 3 and 5 axis machining. Protocols like LSV-2 standardise message exchange over RS232 connections (file transfer, offset data transfer, remote control, etc.). Token Ring (ISO 802.4) and Ethernet (ISO 802.3) interfaces for NC controllers are still quite unusual. Most existing industrial DNC configurations are implemented on the basis of an ethernet network with DNC units (usually based on Personal Computer hardware) which have a serial connection to one or more NC controllers.

\subsection{Limitations of hierarchical sequential communication}

The traditional organisation of manufacturing companies has a mainly hierarchical and sequential communication structure. Information is passed top down only after the function which produced it has completely finished. Because of this, most of the product throughput time is in fact waiting time, which on the one hand makes the manufacturing process rather insensitive to disturbances but on the other hand leads to high inventory cost and long delivery times. In principle, the allocation of jobs to resources is fixed and no alternatives are considered if the resources are (temporarily) unavailable. Feed back information on production progress and process performance is usually not collected or is only used for later statistical evaluation. Production planning controls the manufacturing processes in a unidirectional, hierarchical way. In most cases there are no formal systematic ways to improve the upstream processes by using performance information. Because of this, adaptation of design and manufacturing procedures to changing market demands takes too much time.

\section{The interfaces on factory and system level}

The major problem of design is that the solution domains for design problems are usually very large. Because of this, it is very difficult to consider all the consequences with respect to the total product life cycle. It is hardly possible to check all manufacturing, assembly, maintenance, disassembly and recycling aspects in the early design stages. Unfortunately, during the conceptual design stage the majority of the product costs are committed [2], while the specific aspects mentioned above are mainly taken into account during detailing. Hence, it is very difficult to optimize all of them.

Although most generic manufacturability aspects must be considered by the designer, the flexibility of the manufacturing department in terms of the use of alternative methods and resources should be utilized in order to avoid over-constrained designs. This applies in particular to the problem to predict the availability of specific manufacturing resources ovet time. From many feasible combinations of machine tools, tools and auxiliary equipment, the most suitable set of resources for every order which is released by production planning has to be selected, in such a way that an overall optimum in product quality, due date reliability and cost can be reached.

When orders are accepted, the customer requirements have to be translated by sales into product requirements but also into quality requirements with respect to processes and procedures used. Design and engineering converts the product requirements into part shapes and materials, defines the product structure and specifies the bill of materials. It generates product models which are supplied to Process and operations planning. On the basis of order sizes and, product structure, bill of materials and global resource capacity, Master planning breaks the orders down to batches and attaches internal due dates to them. Quality assurance takes care of the use of proper methods and procedures for the manufacturing and inspection of components and assemblies. It receives the inspection criteria from Design and engineering and gets quality data from Monitoring and diagnostics on cell level. The main task of Process planning is the conversion of product structures, which have been created by Design, into process structure which enables manufacturing and assembly. Process planning supplies Design and engineering with 


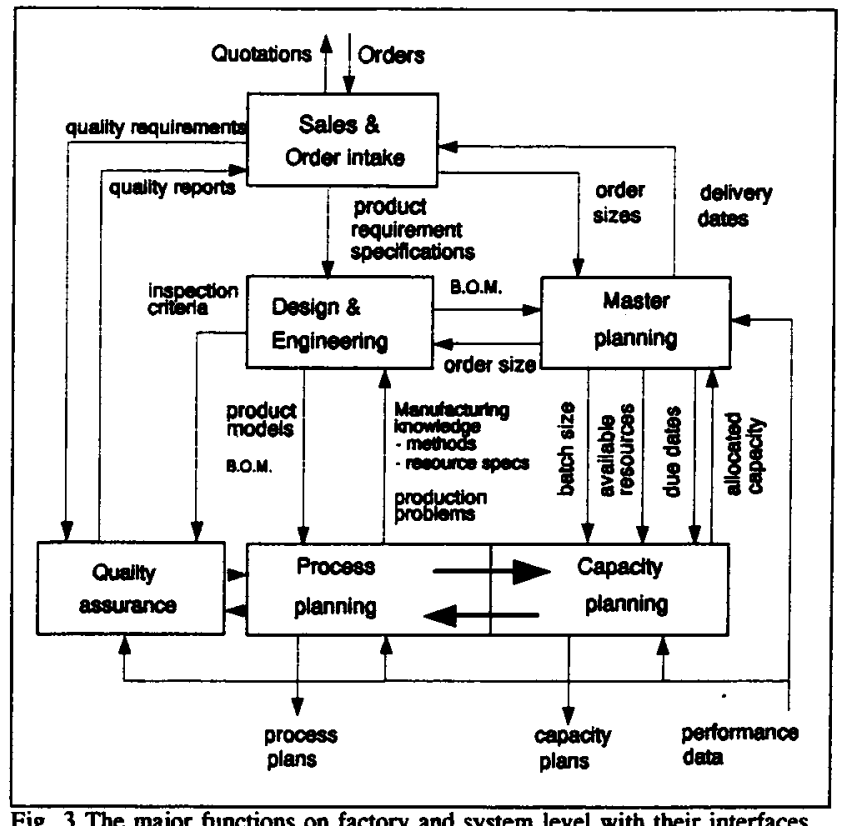

Fig. 3 The major functions on factory and system level with their interfaces

information on available manufacturing methods and resources and feeds back design related production problems. The allocation of manufacturing and assembly resources has to be performed in close cooperation with Capacity planning. Figure 3 shows the functions on factory and system level with their interfaces.

\section{Design for manufacturing}

\subsection{Design methodologies}

In order to make the design process more efficient, several design methodologies have been developed. They can be categorized as prescriptive, descriptive or computational.

One of the pioneers in the field of prescriptive design methodologies was Rodenacker, who introduced a method which was based on the modelling of the function structure and functional properties of a product [8].

A survey on systematic design and formal prescriptive design methodologies is given by Pahl and Beitz [9]. The approach presented in this work is also embodied in the German VDI 2222 guideline for systematic design.

Pahl and Beitz distinguish between the following design phases:

- Clarification of the design task - formalisation of specifications

- Conceptual design - proposition of a principle solution

- Embodiment design - determination of overall layout

- Detailed design - specification of geometry

Suh's prescriptive method, called "Axiomatics" gives a fundamental scientific interpretation of design [10,11]. It comprises a model of the design process, (expressed in terms of functional requirements which are mapped to a set of design parameters) as well as a set of general principles to improve the quality of design solutions. Two Axioms which address the independence of functional requirements and the information content of design solutions form the basis for a number of corollaries which describe general guidelines for good design. The corollaries address issues like decoupling and minimization of functional requirements, integration of design features, the use of standard parts, symmetry, etc. Suh identified four domains in which designers have to work: The customer domain, the function domain, the product domain and the process domain. (In Sohlenius' keynote paper on concurrent engineering, the Axiomatics domains are modified, a process function domain is added which represents the design of the manufacturing system [2]). In the process function domain and the process domain the manufacturing issues are addressed.

Descriptive design methodologies are based on the analysis of observations of experienced designers during their work. Uliman [12] concluded from design protocol studies that designers do usually not follow the sequential order prescribed by Pahl and Beitz but that they continuously move back and forth between the design phases. Usually quite early in the design process, a detailed (partial) solution is chosen, based on a selection from known variants and subsequently an attempt is made to satisfy the other functional product requirements by backtracking and modification. Uliman recorded that designers do pay attention to manufacturability issues already from the very beginning of design process but tend to forget about some of these considerations easily when occupied with functional aspects of the design in a later stage.
Computational design methodologies are mainly based on geometric modelling techniques which are implemented in CAD systems and calculation programs like Finite Element Method, flow analysis, kinematics, etc. The user has to follow specific procedures to create the product geometry and the system supports him with templates and functions which speed up the detailing process.

During the final design stage, the shape, dimensions and (surface) properties of the products components have to be established and recorded in a standard format. Functional aspects like strength, stiffness, weight, center of gravity, moments of inertia, fits and limits etc. are taken into account when determining the actual part dimensions. Standard parts are selected from catalogues. Some designed shapes have to be adapted to that. Conflicts arising at this stage may force loop backs to earlier design stages. These conflicts are often induced by manufacturing and assembly problems.

\subsection{Computer support in the different design stages}

It has been mentioned before that by the decisions to be taken during the conceptual phase the major part of the cost of the final solution is determined. So this phase is very critical and requires a multi-disciplinary overview of all the possible consequences of the design decisions to be taken. The scope and depth of the required problem domain knowledge strongly depends on the kind of design. In the domain of original design, where new function structures have to be developed and existing solution principles cannot be applied, computer programs which stimulate the designers creativity by generating associations might be useful. In adaptive design, where minor modifications are made to existing function structures, computer based design (solution) catalogues can be used. However, there are hardly any computer based support tools for conceptual design available yet.

Computer support is now becoming available for the embodiment design phase. Embodiment design requires systems which can deal with incomplete and abstractly defined geometry of mono-parts and their relations (assemblies). Shapes and tolerances of mono-parts are dependent via the assembly relations. Usually it will be possible to change the shapes of several related mono-parts, (aimed at the resolution of manufacturing problems), without violating the assembly relations of the product model. In this way it is also possible to deliberately define incomplete product models and to postpone design decisions which should over-constrain the product model on arbitrary grounds. Based on AI developments, some commercial embodiment design systems have been released (e.g. ICAD ). They are capable of reasoning about generic objects while satisfying design and manufacturing constraints. Other systems, which are based on more traditional implementation techniques, can be used to create "flexible product models" by using parameterized geometry (e.g. Pro/Engineer and UGconcept ${ }^{\circ}$ ).

Detailed design is the application area of the present generation of commercial CAD systems. They can be very effective if the designer, process planner and NC programmer can sit together at the screen, discussing the manufacturability of designs and coming to compromises. In larger companies with separate departments relying on more formal communication, it is much more difficult to discuss design and manufacturing changes. Because of that, incompatibilities between designs and process will cause expensive delays. The integration of CAD/CAM in large organizations requires elaborate data management. For instance, when different people are concurrently, working on the same product, authorization and version management procedures have to control the release of product models. At present there is an increasing industrial interest in Product and Engineering Data Management systems which allow hierarchically controlled storage of product models for networked CAD/CAM workstations. Larger companies are beginning to put high priority to interfacing their CAD/CAM system to their technical and logistic information systems. Most CAD systems can interface to Finite Element Method analysis systems for stress calculations. However, with respect to detailed design support, the traditional CAD systems are still working in the wrong direction. Shape is the input of analysis tools instead of the output. As a consequence time consuming iteration loops of shape modifications and subsequent analysis have to be made.

\subsection{Feature technology}

Feature modelling is based on the idea of designing with "building blocks". Instead of using analytical shapes like boxes, cylinders, spheres and cones, which are the primitive elements in standard solid modelling packages, the user creates the product model in a better structured way by using higher level primitives which are more relevant to his application. In this way the intents of the designer and the design history can be captured in the product model, which can be very helpful for the downstream tasks.

An engineering feature is defined as: "A physical element of a part that has some specific engineering significance" [13]. It must satisfy the following conditions [14]:

- be a physical constituent of a part

- be mappable to a generic shape

- have engineering significance (meaning)

- have predictable properties 
Features are constituents of parts, which in tum are constituents of assemblies. It must be possible to specify feature attributes, at any level of the product hierarchy, on various levels of abstraction, but, according to the definition above, abstractly defined features must finally become physical constituents of a part whenever the information about them is complete. To complete the definition of a shape, all dimension parameters must be specified. It must be possible to perform various types of computer based and human conducted reasoning on incomplete or abstract feature definitions. However, the relation between form and function is oot formally understood [14]. There clearly is no unique way of mapping function to form and vice versa. A product function can be a result of many interacting sub-functions. It is impossible to define a unique set of one to one relationships between shape elements and sub-functions. Some catalogues with standard solutions to fulfil (sub-) functions do exist [9], but unfortunately they only cover small fractions of the engineering domain.

Features vary from one type of part to another. For instance, many sheet metal part features will not be relevant for machined or composite material parts and vice versa. The composition and decomposition of parts in terms of features varies from application to application [15] (see figure 4). The existence of multiple viewpoints implies the need for multiple concurrent

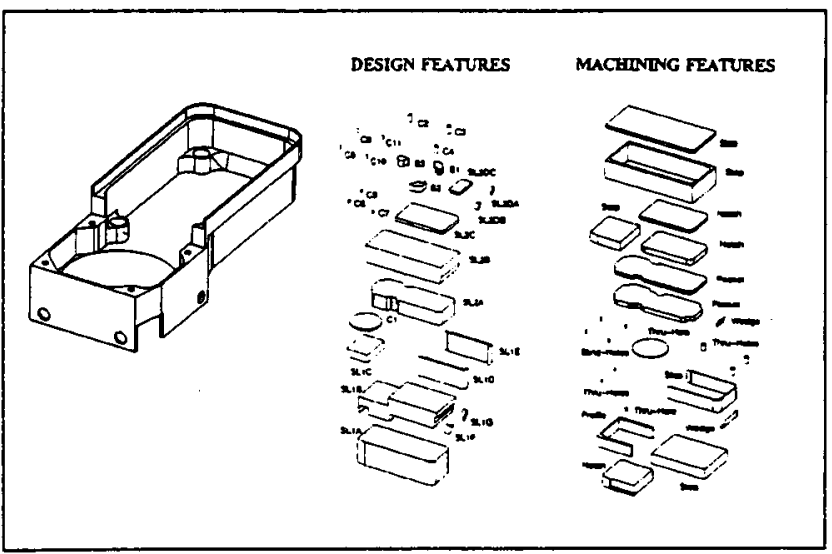

Fig. 4 A Part decomposed in design and manufacturing features

feature models of the same part. Shah has defined the concept of feature spaces which represent collections of features relevant to a specific application domain [16]. Feature spaces can either be partially overlapping or completely disjoint (see figure 5 ). In the conjoint areas, features have identical semantics (for instance, a through bole in machined part has the

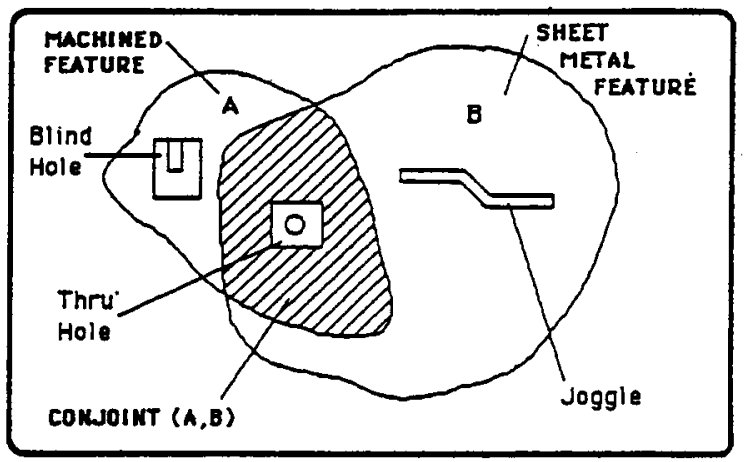

Fig 5. The mapping of feature spaces

same meaning as in a sheet metal part). In the non-overlapping areas, features are oniy meaningful in one domain. The dimensionality of a feature space is determined by the information level which is just sufficient to carry out the tasks in the corresponding domain. Information belonging to one domain may be abstracted to be applicable in another domain. This is called a projection transformation from $\mathbf{n}$ to $(\mathbf{n}-\mathbf{m})$ space. This transformation is unique but the inverse is not, because of insufficient information. Conjugate feature spaces contain features which are composed of different variations of the same elements. Adjoint feature spaces contain associated elements of different nature (such as a load in the analysis space and the boundary on which it is applied in the geometric space). Between completely disjoint feature (sub-)spaces, no mapping is possible. Conjoint (sub)spaces are mapped by the identity transformation. Conjugate feature transformations require (a.o. geometric) reasoning and may include feature extraction, decomposition into lower level entities, reconstruction into different higher level entities, and augmentation with new data or entities.
Because features must be physical constituents of a part when they are completely defined, the basic underlying modelling technique must be geometric. Solid modelling is generally accepted as the technique which must be used for geometric feature modelling. Features can be represented explicitly or implicitly. Implicit (unevaluated or procedural) features are defined only by the description of how to create them and are as such not defined in terms of the primitives of a geometric product model. They are merely defined by their location, orientation and parameters. Explicit (evaluated or enumerative) feature definitions bave been used by most researchers in the field of feature based design and feature recognition.

Features are a very promising tool for the interfacing of design and manufacturing and as such they have the potential to close the gap between CAD and CAPP. Design features must comprise manufacturing aspects and manufacturing features must comprise information on the design intent However, techniques which can map features from one domain to the other must be available.

\subsection{Knowledge based product modelling}

Design comprises a large number of synthesis, analysis and decision making loops. Systems for design synthesis can be classified as rule based systems or learning systems. Rule based systems reason with synthesis rules and generate proposals for solutions. Learning systems are based on catalogue selection in combination with design heuristics. Systerns for design analysis are based on constraint satisfaction, either on an incremental or on a continuous basis Constraint satisfaction can be performed by a truth maintenance system. The combination of these techniques with geometric modelling, constitutes a platform for a new generation of CAD systems which can incorporate manufacturing knowledge [17].

At IVF/KTH a large research program on "product modelling with $\mathrm{Al}^{\text {" has }}$ been carried out from 1985 until 1990. Many different areas were addressed from conceptual design to manufacturing planning. The basic goal was to develop an integrated environment of cooperating systems which could be used to assist designers and process planners in their various tasks $[18,19]$.

General purpose knowledge engineering environments formed the basis for the development of the first commercial "Knowledge-Based Engineering" systems like ICAD ${ }^{\infty}$. Properties of a design, consisting of parts and assemblies can be specified by the use of taxonomies and rules. Manufacturability constraints can be specified as a part of the product model. A "super-user" should first implement the domain classification and specify the problem dependent rules and constraints. He actually must build an application oriented CAD system for the end user, who subsequently can address the system in terms of the application. The performance of the software, the training of super users, the domain classification and the knowledge acquisition are still major problems.

\section{CAD/CAD interfacing}

The exchange of CAD data became an important topic in the late seventies. By that time there were aiready many different CAD systems on the market. The first version of the Initial Graphics Exchange Specification (IGES) standard was released in 1979. It marginally supported the exchange of 2D drawings, usually mutilating their structure and because of that, making them of limited use for further processing on a different CAD system. It was recognised that although the IGES standard was developed further to keep in pace with the developments in geometrical modelling, it was still insufficient for the exchange of complete product model structures.

The German automotive industry developed some CAD interfacing standards like VDA-FS for the exchange of surfaces and VDA-PS for the neutral interfacing of application programs. The latter formed the basis for DIN and later on CEN/CENELEC standards which allow to develop portable standard part catalogues for CAD systems. The catalogues are based on CAD parametric programs and files with standardized part data. Standards are developed so that parametric programs and data files can be developed independently of any specific CAD system and may easily be interfaced to most of the commercial CAD systems. The part data file format is standardized by DIN V-4001 (CEN/CLC draft prENV 40004-24-26) and the way of writing the parametric part programs is standardized in DIN V-66304 (CEN/CLC draft prENV 40004-31). The latter CAD neutral programming interface may be used for a lot of other purposes than the programming of standard parts. An extended version of this programming interface which supports interactive communication with the CAD designer, (the so called BM interface) has been developed at the University of Leuven and is used for all CAD programming purposes, such as writing special design programming software, writing special CAM software, interfacing CAD systems with other application programs, etc [20].

The SET standard was developed by members of the European Airbus consortium. In the USA, the development of PDES (Product Definition Exchange Specification) was started, soon followed by the international counterpart STEP (STandard for the Exchange of Product model data) [21]. STEP supports an extensive set of product model aspects, including form 
features. Because of the wide scope of STEP and because of the large number of people with ideas involved, the standardisation progress is rather slow.

Feature based modelling generates an additional problem with respect to interfacing. The most important dilemma of feature based modelling is that many different methods can be used for the synthesizing of parts from features. This implies that the number of possible features is virtually infinite. It has become clear that features must be user adaptable and that the feature library must be extendable $[22,23]$. However, this can complicate the exchange of product models between different design systems as well as between those systems and other application programs. Agreement must be achieved about a standard set of features and their attributes, but the prescribed use of a fixed set of features will be 100 restrictive. It is not desirable to standardise features as rigidly as in the present version of PDES/STEP. What actually is required is a formal specification language for features $[14,24]$. The European IMPPACT project (ESPRIT 2165) uses a feature definition language called PDGL which is based on EXPRESS, the STEP product modelling language [25]. Future versions of STEP will include user definable features by means of a standardized feature definition language [26].

\section{Computer Aided Process Planning}

Process planning is the act of preparing detailed processing documentation for the manufacture of a piece part or an assembly [27].

Process planning is usually performed in two phases:

1) During the design phase, decisions are being taken about which manufacturing processes are required to produce and assemble the parts. Depending on the type of product; the type of production (order based or non-order based), the lot size, etc. the existing manufacturing resources have to be considered as design constraints or (parts of) a new manufacturing system has to be designed.

2) After the design phase, the individual orders have to be allocated to specific manufacturing resources.

Depending on the production environment the degree of detail in process planning may vary. If it incorporates a detailed elaboration of the individual operations (which is e.g. required for NC machining and robot manipulation) it is usually referred to as process and operations planning. As mentioned before, process planning is partially performed during the design stage, sometimes even before the required manufacturing equipment is installed. In high-volume production facilities, the process plans are virtually incorporated in the composition and layout of the manufacturing system. In the typical instrument makers environment no formal process and operation plans are made at all. If the designer has not violated the constraint of the workshop, the parts can be produced directly from a sketch by experienced machinists. However, in the job shop environment, in which large numbers of small batches bave to be produced, process planning is the indispensable support function which maintains the information flow. Usually process planning for the job shop environment requires a high level of detail because of the frequent set up changes which are typical for small batch manufacturing.

Process and operations planning comprises [28]:

\author{
- the interpretation of the product model \\ the selection of machine tools \\ the selection of tool sets \\ - the determination of set-ups \\ - the design of fixtures \\ - the determination of machining methods \\ - the selection of tools \\ - the determination of machining sequences \\ - the calculation of tool paths \\ - the calculation of process conditions \\ - the generation of NC programs \\ - capacity planning
}

Usually the selection of the most appropriate processes, machine tools, fixtures, machining methods and tools can not be performed in a fixed sequence because of mutual dependencies between manufacturing entities and the impossibility to evaluate feasible solutions in full detail in the early stages of the planning process.

Each of these functions in its own represents a complex process of generation and comparison of alternatives. Many of the decision making processes are interdependent. During actual production there is little time available for the selection and preparation of machine tools, tools and fixtures and for the generation of (configuration specific) NC programs. The process planning activities have either to be performed on beforehand or have to be accelerated in order to meet the real-time requirements of on-line process planning.

The major characteristic of process planning for fixed manufacturing environments is the combinatorial nature which can be expressed in terms of a manageable set of facts and rules which can be used to map product geometry to manufacturing operations. A complication is the limited capacity of the manufacturing resources and the unpredictability of their actual availability on beforehand if many concurrent jobs are competing for resources. In order to achieve an acceptable level of flexibility it is absolutely necessary to have some freedom in selecting alternative processes and operations. Only in this way the due dates can be met.

\section{Features as the interface between CAD and CAPP}

In current process planning praxis, the product model analysis process is usually not performed in a systematical way. The same product model can yield quite different process plans when dealt with by different process planners. Even the same planner may have difficulties in producing exactly the same plan if he had to do it twice. This causes an unwanted diversity in methods, routings and tools. By the use of handbooks and instructions, companies try to rationalize process planning. The method of archiving process plans as templates for future variants and the use of standard machining features (macro's), for which fixed sets of machining solutions are specified, reduce the diversity. However, because of the large product variety in small batch manufacturing, the applicability of templates is limited and it remains a tedious work to retrieve the features from the product model manually. Some attempts have been made to automatically recognize features from 2D drawings but with limited success. Because of the fact that very little CAD/CAM systems, which are currently in use in industry, do support solid modelling. some successful implementations for automated process planning and machining of prismatic components have been developed on the basis of 2.5D wire frame models, for instance at UMIST. However, full 3D solid models are to be preferred for effective feature based design and manufacturing because of their unambiguity and the availability of surface information.

In principle there are three different methods to apply manufacturing features [14]:

- Design with manufacturing features

- Retrieval by interactive definition

- Retrieval by automatic recognition

\subsection{Design with manufacturing features}

Design with manufacturing features is used if the part geometry can be completely defined as a composition of standard manufacturable objects. Feature based design allows the designer to specify much more about the design than just the geometry and the topology. The design intent and the constraints can be expressed on different levels of abstraction and can be used in downstream applications, even in an incomplete form. However, a serious disadvantage is that the designer is forced to think mainly in process planning terms. He is limited to some collection of very specific "manufacturing building blocks". Interactions between manufacturing features have to be analyzed thoroughly with respect to position and tolerance relations and to (economic) manufacturability of the resulting feature combinations [13]. Manufacturing feature based modelling systems without adequate geometric modelling capabilities have proven to be unacceptable. They can create nonsense geometry and do not guarantee unambiguity.

The set of features required for a general feature based design system is virtually infinite. The creation and maintenance of an adequate library of features with an appropriate level of abstraction forms a major threshold for feature based design. However, if features are user definable, specific feature based design systems are feasible. Since many features are application specific and have to be mapped to (generic) manufacturing processes and resources, the need for feature mapping or recognition does not implicitly disappear by the use of feature based design [14].

\subsection{Interactive definition of features}

Interactive definition of features can be performed by picking and grouping geometric elements from a product model representation on a screen in such a way that the groups adhere to the definition of a standard manufacturing feanure for which manufacturing methods have been defined. This information can subsequently be extended with entity attributes such as radii, depth, tolerances, surface finish and higher level attributes center-line position, orientation and distance etc. This method can easily be applied to Boundary-representation (B-rep) solid modellers and it has been used by many researchers to generate input for their process planning and NC programming systems $[29,30]$. The disadvantage of the method is its labour intensiveness and its sensitivity for human errors.

\subsection{Automatic recognition of features}

Automatic feature recognition comprises many different, partially overlapping methods which all are based on searching the database of the modeller for matching patterns (topology and geometry) and extracting the feature parameters from them. Only some recognition techniques are able to combine simple features to hierarchies of complex features [31] but for most prototype implementations the recognition of intersecting features remains a big problem. The set of features to be recognized has to be user-extendable in order to be able to adapt the recognition algorithms to new shape elements. 
However, it makes no sense to recognize features for which no manufacturing methods have been defined. In this sense, the (theoretical) completeness problem, which has been the major point of criticism on feature recognition does not exist.

At present, feature recognition undoubtedly is the most versatile technique for the transformation of product models between application domains. Because the design history is not recorded in the current generation of CAD systems, and only the final result of the design process is available for the downstream applications, feature recognition has the important advantage that it can provide the proper information to those applications, independent of how the product model was created. Additional to research on recognition of explicit features, the mapping of implicit and abstract feature are areas on which many projects will be carried out in the near future.

\section{Existing implementations of feature based interfaces between CAD} and CAPP

\subsection{Generative CAPP}

It has become clear that CAPP systems which are purely based on storage and retrieval of (variant) process plans are not fit for use in an NC environment. Generative CAPP systems synthesize process information from models and rules in which the manufacturing logic is stored. The term "generative" relates to the fact that a process plan can be generated from a workpiece description with little or no human intervention and without using templates of look-alike products. Most generative systems are capable of performing process sequencing, tool selection, cutting conditions etc. automatically. However, the generation of cutter path information requires complete geometric models (Group Technology codes are not sufficient for this level of detail).

The CIMS/PRO process planning system [32] in combination with the CIMS/DEC part description system [33] can handle linear and rotational sweeps of concatenated 2D profile elements which can carry technological attributes. Although the description of the part domain is limited, the systen represents one of the first attempts to create an automatic feature based link between CAD and generative CAPP. TIPPS [29] is another example of a system with a such a link to CAD.

\subsection{PART}

An example of a generative CAPP system which meets most of the identified manufacturing interfacing requirements is PART [31] (an acronym for Planning of Activities, Resources and Technology). The system has been designed to achieve a very high level of automation in the planning of processes and operations which can be performed on machining centres. PART can derive (sets of) geometrically, technologically and economically correct NC programs and related documents from (B-rep) product models fully automatically. PART recognizes manufacturing features from the B-rep model on the basis of a set of user extendable feature patterns and allocates machining methods and tools to them. It selects the most appropriate machine tools, set-ups and fixtures and manufacturing methods on the basis of a knowledge base which is user adaptable in a graphical interactive way. PART automatically determines tool paths and cutting conditions and calculates machining times and costs. Production orders are allocated on the basis of suitability and availability of machine tool capacity. Technological planning decisions are not only subjected to technical process constraints but also to capacity and availability constraints of the resources. The system is fully configurable, as mentioned before, features, manufacturing methods,

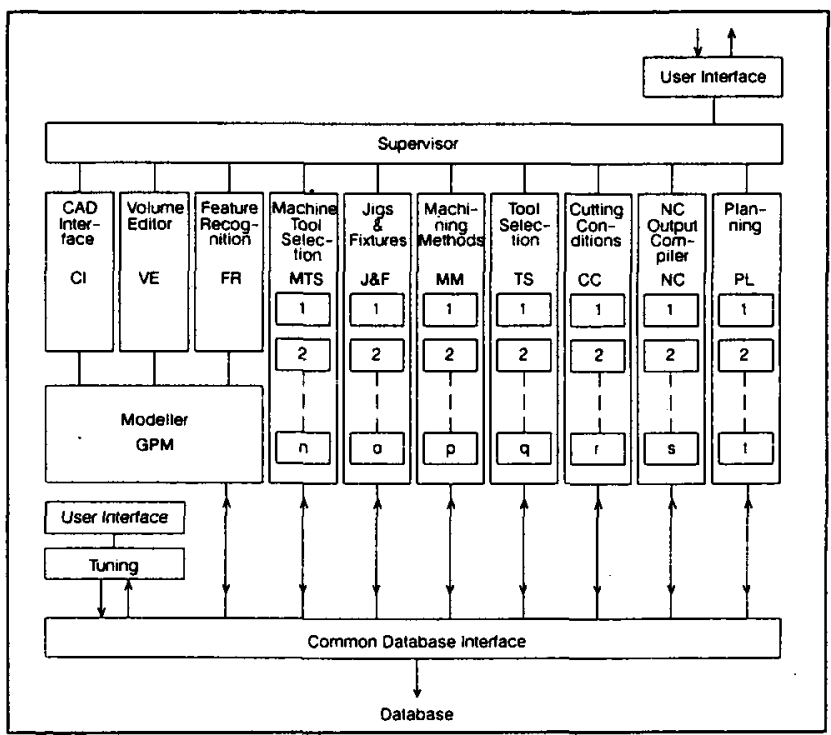

Fig. $6 \mathrm{~A}$ schematic representation of the PART system. optimization strategies and the sequence of process planning decisions can be adapted and/or extended. Figure 6 shows a schematic representation of the PART system. The product model is imported via a STEP interface. After decomposition the features remain linked to the geometric product model, which is important for collision checks and NC path optimization. The feature hierarchy, which is retrieved automatically, is used by the manufacturing method selector module for the combination and the evaluation of precedence relations of operations. Machining conditions are optimized for the selected combination of resources. The system is capable of generating complete process and operation plans quick enough to be applicable as an online process planning system. The PART system consists of a large number of "phases", which can be executed according to user definable "scenarios" under control of a supervisor program[34]. The system's behaviour can be tailored to the preferences and priorities of the company. With appropriate scenarios the PART system can also be used to perform manufacturability and cost calculation of parts in the design stage.

\section{The interfacing of CAPP with capacity planning and quality assurance}

The main objective of CAPP is to find the best way to materialize a given set of products within the constraints of the manufacturing resources. Overloading must always be avoided. If capacity planning has no proper interface to process planning, the workload of the individual machines will become unbalanced. Optimization on a pure technical basis will lead to the allocation of the majority of the jobs to those machines which have the best price-performance ratio, while the utilization rate of other machines will be low. Figure 7 gives an overview of the relations betwien Process planning, Capacity planning and Quality assurance.

\subsection{Capacity planning}

A capacity plan contains all the information which is required to manufacture a planned volume of products within a given period of time. Capacity plans are the basis of shop floor control. Capacity planning must take into account the relations between the different jobs. Jobs can have prescribed and/or preferential sequences. Prescribed sequences result from the product structure and preferential sequences are based on the optimization of the utilization of resources.

\subsection{Quality assurance}

Quality assurance has to validate the methods and procedures which are used

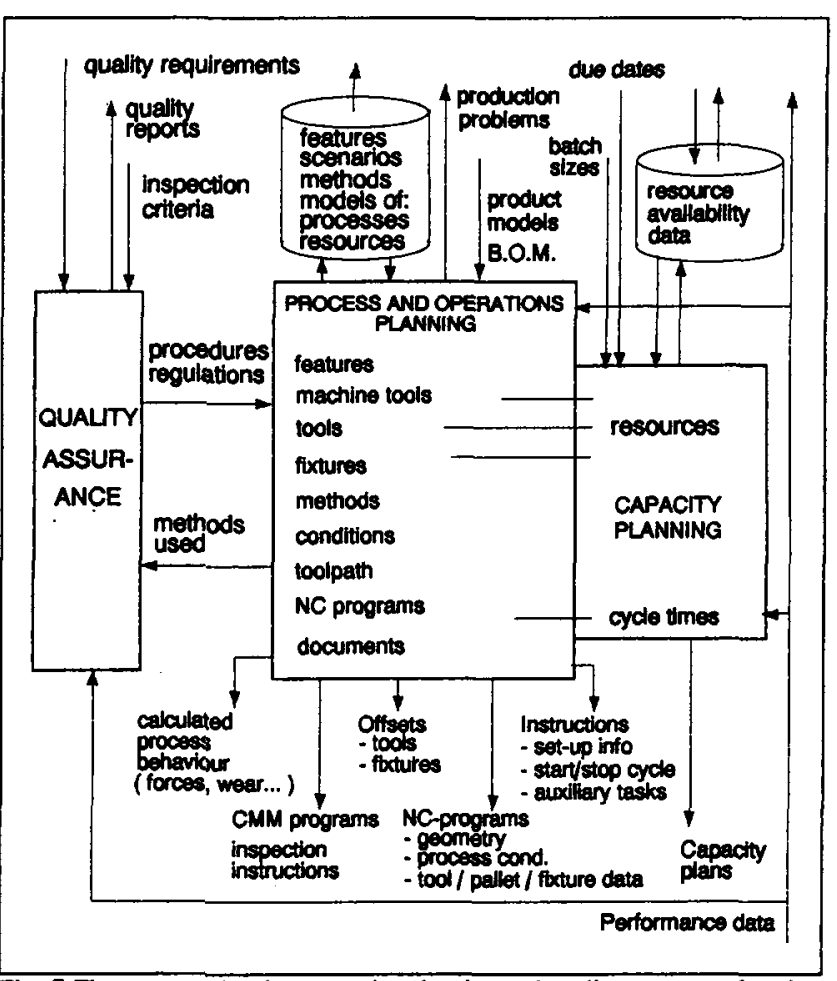

Fig. 7 The process planning, capacity planning and quality assurance function and their interfaces

for manufacturing and assembly. It receives inspection criteria from Design and passes regulations and inspection procedures to process planning. On the basis of this information, quality control instructions and for instance, the programs for Coordinate Measuring Machines (CMM) can be made. Process planning reports its working procedures and quality problems back.

13.3 Re-allocation of jobs 
It is rather difficult to allocate all the individual jobs, which are comprised in the capacity plan, to the available resources of the workshop in such a way that all the processes run economically and all the due dates are met. However, it is even more difficult to cope with rush orders and break-downs. If process planning is only an off-line activity, it is impossible to react sufficiently quick on unexpected disturbances. Usually disturbances cannot be dealt with by just re-arranging the schedule. When jobs are re-allocated to other machine tools, which also may involve the need for other tools, severa adapted or completely new process plans are required almost instantaneously. The higher the capacity utilization, the higher the risk that a small disturbance will propagate and cause many additional problems before the original problem can be solved. This avalanche effect can only be avoided if the capacity plan provides some backlash between subsequent operations, if the response time of the system is sufficiently short and the solution itself is adequate. This implies that the degree of capacity utilization is an important tuning parameter of the manufacturing system.

\subsection{Alternative process plan}

In order to prohibit propagation of disturbances it is vital that a situation can be analyzed quickly and that alternatives are provided on a real-time basis. The first problem which must be solved is, whether the actions to be taken will simplify or complicate the situation; in other terms, if a re-allocation of jobs is beneficial. If the disturbance causes only a relatively short delay, may be better just to wait and shift all the activities of the work plan accordingly. This solution may cause less problems than re-allocation of jobs. If jobs do have to be re-allocated and new process plans must be provided, there are three possibilities:

-To generate a number of alternative process plans on beforehand.

-To generate a "non linear" process plan. $[35,36]$

-To generate alternative process plans instantaneously (at run-time).

The first possibility is applicable to process planning in the design phase. Alternative (generic) manufacturing and assembly solutions should be considered. However, if the number of orders is large, too much redundan work is created if all alternative process and operation plans have to be elaborated. Because of the usually large number of feasible combinations of machine tools, set-ups, machining methods and tools, it is difficult to estimate what the probability is that a given alternative will ever be used

The second solution has a similar problem as the first one. The probability that a process plan can be applied increases with the number of alternative "routes" in the plan. However, the time which is required to create non-linear process plans increases with their complexity. The more complex the net, the more difficult it becomes to find an alternative "route" through the plan a run-time. An additional problem is that alternative (partial) NC programs need to be generated because NC programs usually are not exchangeable between machines.

The third solution is specifically applicable to order based production with (more or less) fixed manufacturing resources. It avoids redundant work, but i is only feasible if alternative generic plans can be elaborated sufficiently quick, which is only possible if the level of automation in process and operations planning is very high. When generating alternatives, the system must have access to the data which exactly reflect the availability and the actual work load of the resources.

\subsection{On-line process planning}

Adaptations of original process plans must be scrutinized for side effects. It is very important to avoid propagation of disturbances. A short delay of a few jobs may cause little extra cost, while a too rigorous re-allocation of jobs may result in a dead-lock situation. A "crisis" work plan must diverge as little as possible from the original one. This implies that it is much more important to investigate what can be left unchanged, than to concentrate on the generation of an "optimum" revised work plan. Because of this, the constraints applied to process plans which are generated off-line must differ from the constraints applied to those parts of process plans which have to be modified on-line. This complicates the task of the process planner as he has to switch between two modes: The off-line "optimization" mode and the on-line "trouble shooting" mode. Only generative process planning systems have the potential to solve these problems. However, they must operate in a highly automatic mode in order to be quick enough and they must have access to dynamic shop floor data in order to be able to generate a realistic work plan. Recently developed CAPP systems like PART [31] are capable of performing on-line process planning for complex workpieces.

The interfacing between process planning functions and other manufacturing functions gradually becomes of more interest in CAPP research. For instance, Iwata recently proposed a concept for a more tight integration of CAPP with scheduling [37].

\section{The interfaces on cell level}

The number of installed FMS's increased much slower than was expected but some of the control concepts for FMS appeared to be useful for the control of Flexible Manufacturing Cells. As mentioned before, FMC's are loosely integrated, usually not fully automated configurations of NC machine tools and auxiliary equipment which are connected to a higher level control system. Various supervisory control concepts for FMS and FMC have been proposed. In general, FMC requires a much more flexible control structure than FMS because of the different levels of automation of its constituent workstations and the inherent cycle time insecurities. The control concept for FMC is usually referred to as Shop Floor Control (SFC). Arentsen has conducted an extensive survey on concepts for SFC [38].

The ESPRIT project 809 research project developed a SFC system with a system architecture which is compliant to the three lower levels of the factory model presented in this paper [3]. Since one year, the system is fully operational and in every day use at a pilot company in the Netherlands.

\subsection{The interface between CAPP and Shop floor control}

SFC systems incorporate functions such as scheduling, disparching, monitoring and diagnostics on the cell level but also workstarion control and auxiliary station control on the workstation level. The feed back of information about the situation on the shop floor in terms of availability of resources and performance of the manufacturing processes is absolutely vital for the creation and improvement of realistic process and operations plans. Breakdowns and rush orders can make process plans invalid. Re-allocation of jobs to other resources may sometimes be necessary. This requires the possibility of on-line adaptation of process plans and quick re-generation of NC data. From this point of view, a SFC system represents the interface between CAPP and the production equipment.

Capacity plans and process plans serve as an input to SFC. The system combines the different process plans according to the capacity plan for a specific production period into a workplan, which consists of many different production jobs. A job is defined here as a set of operations which is allocated to a workstation. The shop floor control system schedules the jobs and takes care of the execution of the process plans by releasing the jobs and related auxiliary tasks. A choice of different scheduling strategies may serve different objectives in the control of the material flow, such as first come / first serve, due dates, maximum utilization of resources, etc. Real-time shop floor control starts with the dispatching of the preparatory tasks, which have to precede the actual execution of a production job (e.g. the assembly and presetting of a tool set, the preparation and alignment of a fixture, the loading of part programs and offsets and the transmission of operator instructions). The manufacturing process can only start after all preparatory tasks have been reported ready. The execution of the jobs is monitored and discrepancies between the schedule and the actual situation on the shop floor is diagnosed. The results of the diagnosis are fed back to the scheduler but also to the functions on system level. For instance, the validity of the estimates of the job cycle time and process behaviour are fed back to master production planning, capacity planning and process planning. The dispatcher distributes the NC programs and related information and releases the jobs for production.

14.2 The shop floor control functions on cell level and their interfaces

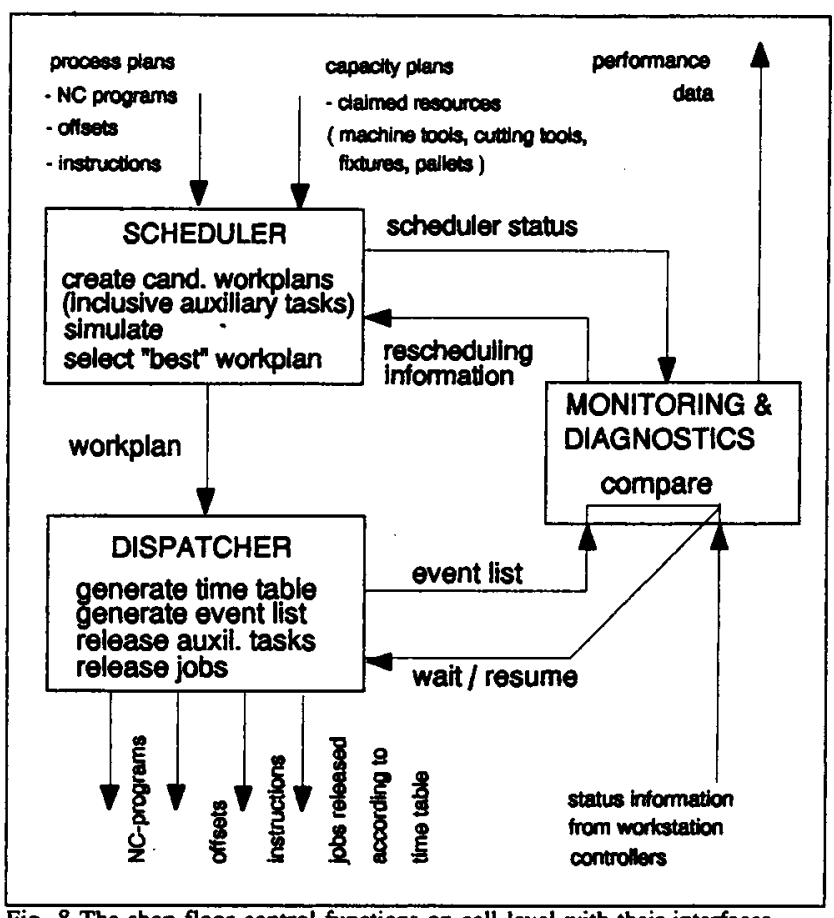

Fig. 8 The shop floor control functions on cell level with their interfaces 
The shop floor control functions which are located on cell level and their interfaces are shown in figure 8

Scheduling can be carried out with the help of a simulation program which generates a number of candidate work plans. Quality parameters are assigned to each candidate work plan. Examples of quality parameters are: The number of jobs exceeding the due date, the make span, the utilization of workstations, the idle times of the workstations and the tardiness. The best work plan among the candidates is selected. Scheduling is usually initiated when the current work plan is almost finished. The scheduling module can also be used to investigate the consequences of the disturbances on a work plan. The re-allocation of jobs to other workstations may invoke severe side effects and can lead to chaos. Usually it is better to accept delays if they are expected to be relatively short.

The Disparcher releases jobs and auxiliary tasks to the different workstations. It compares the scheduling information which is contained in the work plan with the real-time shop floor status information which is supplied by Monitoring and diagnostics. If the Disparcher notices the delay of main or auxiliary tasks which might affect jobs which already have been released, this is reported to Monitoring and diagnostics. If the expected delay is substantial this information is relayed to the Scheduler.

Monitoring and diagnostics collects status information from the workstations and supplies it in an appropriate format to the Scheduler and the Disparcher. It can display both the planned and actual production progress on the scheduling screen in Gantt charts or performance histograms. The data is stored in the cell database which allows later consultation and analysis by the cell supervisor. Monitoring and diagnostics also reports data on the performance of the cell to the system level.

After the jobs have been released to the workstations, their progress is monitored. In the case of disturbances, which may lead to discrepancies between the planned status and the actual status of jobs, the severity of the problem is analyzed. If necessary, the cell controller undertakes actions in order to solve the problem.

On the cell level, also a set of support functions is located. They comprise the resource databases and editing facilities. As an example, the tool information system is elaborated. In the Tool database, all data on individual tools, tool assemblies, tool sets and tool locations are stored. Tool data can be retrieved for process planning purposes and composed tool sets with their gauge lengths can be stored. If required tools are not available on a long term

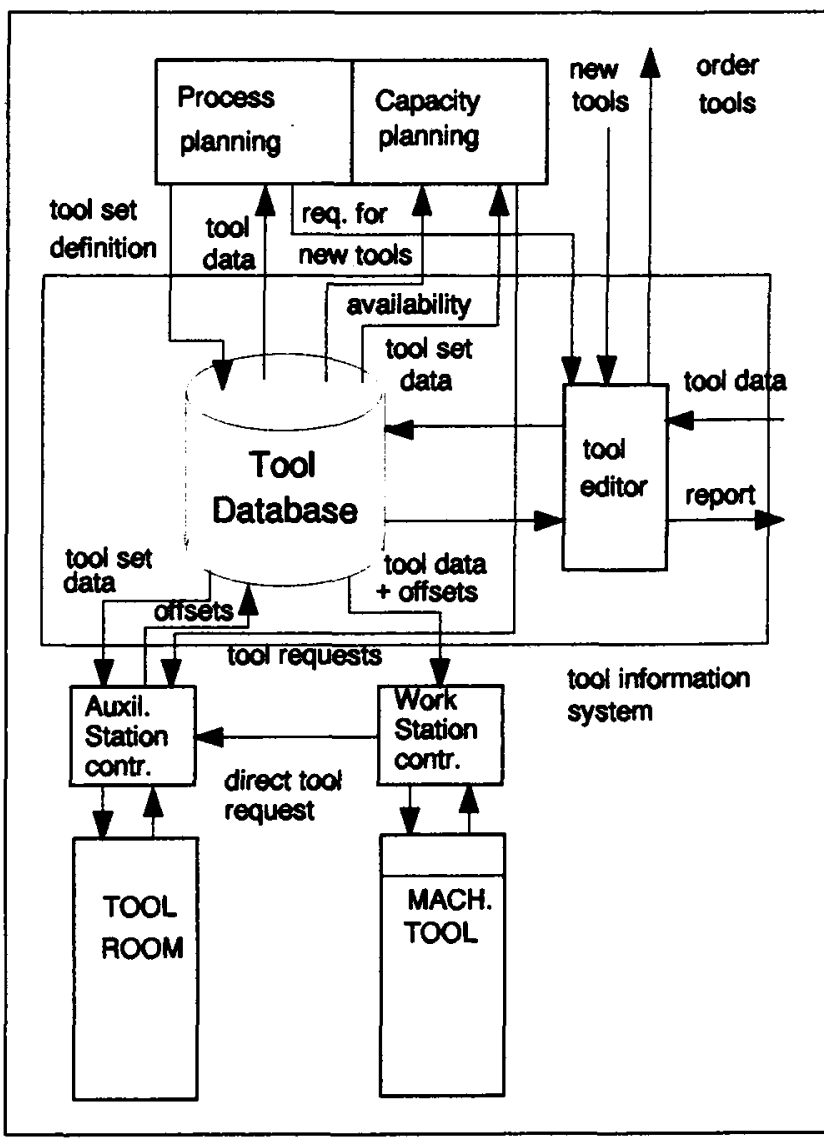

Fig. 9 The tool information system and its interfaces

basis, process planning can issue a request to the tool manager, who can order new tools through Purchasing. The data describing the new tools is entered via the Tool editor. The Tool editor can also be used to generate reports on tools in the database. Capacity planning consults the database for the availability of tools during specific production periods and retrieves data on the composition and location of tool sets. It issues tool requests to the Auxiliary station controller of the tool room (via the Scheduler and Dispatcher). The tool room reads tool set data and gauge lengths which have been specified by process planning from the database and retums offset values after presetting. On schedule, the tool set data and offsets are send to the respective workstation controllers by the Disparcher. In the case of a tool related disturbance like breakage, a workstation can issue a Direct Tool Request to the tool room. Data on the other resources, like fixtures, pallets and materials, can be dealt with in a similar way. Figure 9 shows a diagram of the tool information system and its interfaces.

\section{The interfaces on workstation level}

On the workstation level the workstation and auxiliary control functions are located. Figure 10 shows the workstation controller with its interfaces to the cell and equipment level functions. The workstation controllers coordinate and monitor the execution of the jobs which are allocated to it.

The functions of the workstation controller include:

- Down loading of part programs, presetting data (offsets of tools and fixtures).

- Distribution of control commands to the equipment controllers (start/stop cycle etc.)

- Monitoring of the status of the equipment and the progress in the execution of the jobs.

- Down loading of setting data for the process monitoring equipment.

- Collection and filtering of process monitoring data.

- Filtering of workstation status and process performance information before it is sent to the diagnostics module.

- Serving as a shop floor terminal for the operator.

In the ESPRIT 809 implementation, the design of the workstation controller is based on the so called Virtual Workstation Concept (VWC). This implies that the different workstation controllers in the cell show exactly the same behaviour from the point of view of the cell controller, independent of the degree of automation of the equipment. They control the execution of the jobs by addressing the equipment controllers and, if necessary, also the workstation operator. A workstation controller can supervise several equipment controllers and hence a workstation can vary from a conventional machine or a simple manual assembly station to a complex configuration of

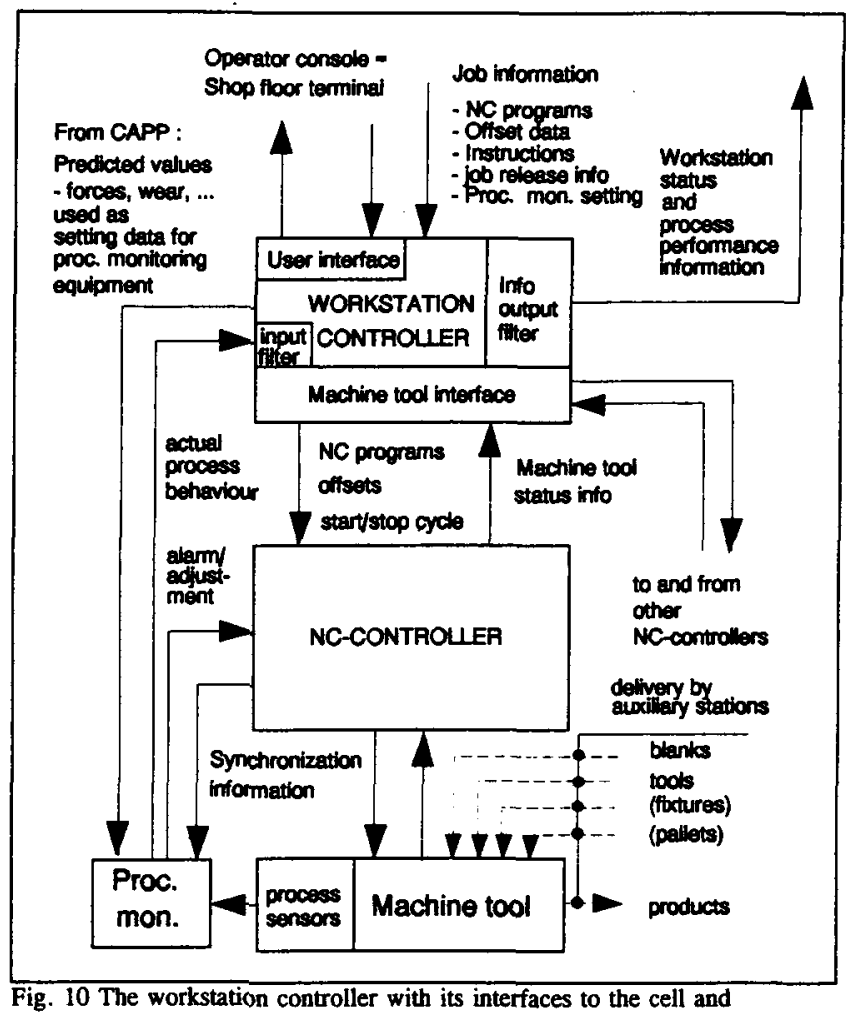
equipment levels

automated machines, handling and transport devices. The workstation controllers are implemented on standard PC's. In the present implementation at a pilot company the workstation controllers are connected to the cell level computers via ethernet. The connection to the different equipment controllers varies between BTR and LSV-2.

16 The interfaces on equipment level 
On the equipment level the physical manufacturing and assembly processes take place. Also the storage, transport, handling, presetting and quality inspection functions are located here. In the typical small batch FMC environment, most of the latter functions will not be fully automated. No automated functions are activated via messages on shop floor terminals Depending on their complexity, the automated handling and transport functions are either controlled by separate auxiliary station controllers or

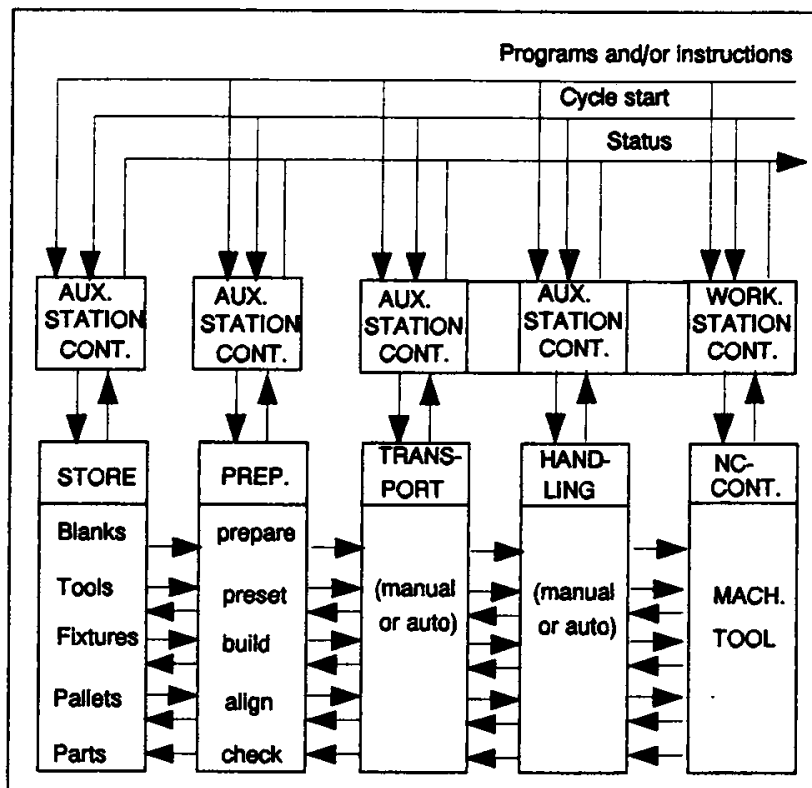

Fig. 11 The main and auxiliary functions on equipment level

directly via the controlier of one of the main workstations.

\subsection{Process monitoring}

Process monitoring can be either be performed by the NC-controller itself (if it is equipped for it) or by separate devices, which have to be programmed and synchronized with the NC program. Predicted values of important process parameters which are calculated by process and operations planning are extracted from the job information and loaded into the process monitoring devices. Data collected by the process sensors is referenced against the predicted values and in case of discrepancies, alarms can be triggered or process conditions can be adjusted (real-time adaptive control). The same data can be used for feed-back to the higher levels after appropriate data reduction. This information can for instance be used by process planning for the correction of process models (non real-time adaptive control).

\subsection{Tool management}

Tool management is an activity which usually is centralized and which needs an auxiliary station controller. The assembly of the tools is performed manually but usually computerized tool presetting equipment is used. The controller of the presetting equipment is interfaced to the tool station controller, by which the tool room operators get their instructions for the tool preparation jobs. The nominal tool gauge values, which are defined by process planning are loaded into the presetting equipment and the actual offset values after presetting are loaded into the NC controller of the respective machines. Other preparatory functions, like fixture construction and presetting and part quality inspection can be interfaced in a similar way. Figure 11 shows the storage, preparation, transport and handling functions on equipment level with their controllers on workstation level.

At KTH, research in this field has led to the conclusion that data communication in SFC's can be simplified and made more reliable by linking the data to the physical objects to which they relate (tools, fixtures, etc. with programmable memory). The principle has been demonstrated a.o. by SMTSajo at the Hannover fair in 1991 . However, this does not eliminate the need for a supervisory system which keeps track of the manufacturing resources.

\subsection{Inspection of the part geometry}

If the inspection of the part geometry and surface quality is performed with automated devices such as Coordinate Measuring Machines CMM programs and operator instructions have to be supplied via an auxiliary station controller. Discrepancies between the design geometry and the actual product geometry which are caused by tool wear or presetting errors can be converted into new offset values which can be used in rework operations. These corrections can be fed back to the machine tool if the job is still active or to the tool room in case the job has already been finished. The number of parts which need rework or are rejected can be reported to cell and system level, in

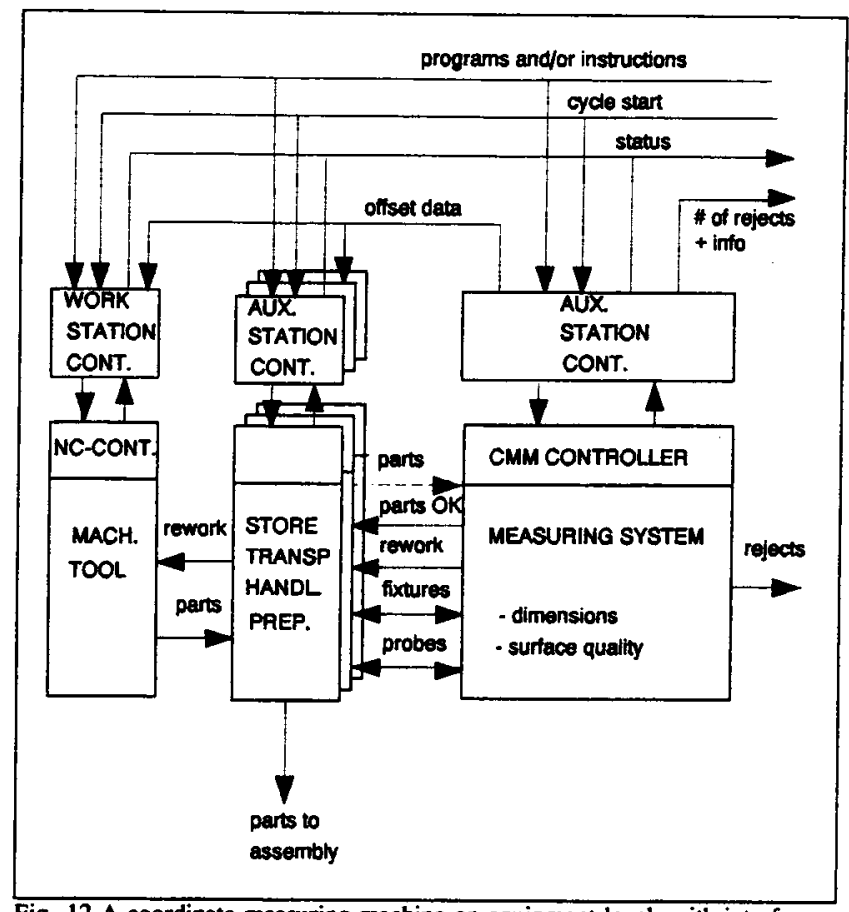

Fig. 12 A coordinate measuring machine on equipment level, with interfaces

order to take action with respect to additional material which might be needed and the expected delay of the job. Figure 12 shows a diagram of the interfacing of CMM to the shop floor control system.

\section{Conclusions}

The material presented above is the result of many interesting discussions in the working group on "Manufacturing Interfaces". An attempt has been made to identify the main functions in small batch manufacturing (including design and assembly) and to put them in a framework which shows their most important interfaces. The diagrams presented in this paper are by no means complete with respect to the details. They should be regarded as a framework for further discussions. Many interesting techniques for the modelling of companies, control systems and resources are under development and a whole range of formal specification methods and structured design techniques are currently available. They have not been used or discussed this paper, because of its rather wide scope and the superficial level of detail. More details on Manufacturing Interfaces can a.0. be found in [39].

At present, most of the identified interfaces have been implemented in some form at different laboratories and companies. However, many implementations are proprietary and only prototypes do exist. Standardization of most of the interfaces is not yet possible because there is no large scale agreement about how the products and manufacturing systems should be modelled, about which functions should communicate, how they should communicate, about the content and rate of the information exchange and about the way product and process data can be stored and retrieved.

CIRP is not the institution which should spend a lot of effort on the development of standards, but its members should be aware of existing and lacking interfacing standards. Besides that, discussions within CIRP about modelling and control of technical and logistic processes and the resulting communication requirements should result in providing input to the standardisation bodies via the individual CIRP members.

The concepts of feature based design and feature based manufacturing represent the most promising interface between design and process planning and can be considered as the real first step to concurrent engineering. However, many problems in the areas of feature specification and feature mapping have still to be solved. A higher level of integration between design and process planning must be achieved. This could be an area of cooperative work between members of STC "Dn" and (the CAPP working group of) STC "O".

A high level of automation of process planning enables quick reallocation of jobs to manufacturing resources and hence increases the flexibility of the work shop. It also avoids the propagation of disturbances. A tight coupling between process plainning and capacity planning leads to realistic schedules which can be met in practice. This makes the simation on the shop floor less hectic, which has a positive effect on due date reliability and product quality. The integration of technical and logistical planning of manufacturing and assembly processes could be an interesting topic for cooperative work within STC"s " $A$ " and "O". 
The typical generic environment where the presented reference diagrams apply are Flexible Manufacturing Cells. This type of environment is found in the majority of small batch manufacturing companies all over the world. The use of computer based shop floor control systems makes it possible to run many concurrent tasks in partially automated small batch manufacturing systems on time without loosing the overview. Feed-back of the actual situation on the shop floor in terms of production progress and process performance improves controllability on the short term and overall system performance on the long term. The enhancement of equipment controllers with higher level programming facilities, improved communication capabilities and interfacing with process monitoring equipment for adaptive control are topics for cooperation between members of STC's " $A$ ", "M" and "O".

The working group on Manufacturing Interfaces will continue to function as a catalyst for continuing discussions on these topics.

\section{References}

[1] Alting, L., Contribution to Round Table, Annals of CIRP, Vol 39/2, 1990

[2] Sohlenius, G., Concurrent Engineering, Keynote paper at the 42nd General Assembly of CIRP, to be published in Annals of CIRP, Vol41/2, 1992

[3] Tiemersma, J.J., Kals,H.J.J.: A Real-time Monitoring and Control System for Small Batch Part Manufacturing. Proceedings of the Manufacturing International ' 90 ASME Conference, Atlanta, USA, 1990.

[4] Kals, H.J.J., van Houten, F.J.A.M., Tiemersma, J.J., CIM in small batch manufacturing. Proceedings of the 22nd CIRP International Seminar on Manufacturing Systems, Enschede, 1990.

[5] Kals, H.J.J., van Houten, F.J.A.M., On Flexible Manufacture based on a Production Information Management System, Proceedings of the 14th CIRP International Seminar on Manufacturing Systems, Trondheim, 1982

[6] van Houten, F.J.A.M., Tiemersma, J.J., An adaptive control module for ROUND, Proceedings of the 19th CIRP International Seminar on Manufacturing Systems, Penn-state University, 1987.

[7] DeVries, M.F., Duffie, N.A., Kruth, J.P., Dauw, D.F., Integration of EDM within a CIM environment, CIRP Annals, Vol.39/2, 1990

[8] Rodenacker, W., Methodisches Konstruieren, Springer Verlag, Berlin, 1971

[9] Pahl, G., Beitz, W., Engineering Design, a Systematic Approach, Springer Verlag, 1984

[10] Suh, N.P.,Rinderle, J.R., Qualitative and Quantative Use of Design and Manufacturing Axioms, Annals of CIRP, vol. 31/1, pp 333338,1982

[11] Suh, N.P., The Principles of Design, Oxford University Press, New York, 1988

[12] Ullman, D.G., Dietterich, T. G., Stauffer, L., A Model of the Mechanical Design Process Based on Empirical Data, Artificial Intelligence for Engineering, Design, Analysis and Manufacturing, vol. 2, 1988.

[13] CAM-I, Functional requirements for a Feature Based Modelling System, CAM-I R-89-GM-01, Arlington, 1989.

[14] Shah, J.J., Philosophical development of form feature concept, in Proceedings of the CAM-I features symposium, P-90-PM-02, 113-128, 1990.

[15] Hummel, K.E., The role of features in computer aided process planning, Proceedings of the CAM-I features symposium, P-90-PM-02, 1990.

[16] Shah, J.J., Feature transformations between application specific feature spaces, CAE joumal, 5, no. 6, 1989

[17] Kimura, F., Suzuki, H., Variational product design by constraint propagation and satisfaction, Annals of the CIRP, vol35/1, 1986.

[18] Kjellberg, T., Product model knowledge bases, Project plan, Dept. of Manufacturing Systems, Royal institute of technology, Stockholm, (in Swedish), 1985

[19] Kjellberg, T., Tools for intelligent human communication and collaboration for better manufacturing, 2nd Toyota conference: Organization of engineering knowledge for product modelling in CIM Elsevier, Tokyo, 359-379, 1988.

[20] Kesteloot, P., Belaen, S., Kruth, J.P., System independent programming interface, Final report of development group MO, BRITE-MODESTI project 1391, Mould Design and Manufacturing Optimization by Development,' Standardization and Integration of CAD/CAM Procedures, 1990.

[21] Standard for the Exchange of Product Model Data, ISO/TC184/SC4 Working Draft of Version 1.0, 1988.

[22] Shah, J.J., Rogers, M.T., Functional requirements and conceptual design of feature based modelling systems, Computer Aided Engineering Journal, 5, 9-15, 1988

[23] Shah, J.J., Rogers, M.T., Feature based modelling shell: design and Implementation, in Procedings of the ASME Computers in Engineering conference, San Francisco, 1988

[24] Pratt, M., Automated feature recognition and its role in product modelling, Proceedings of the CAM-I features symposium, P-90-PM-02, 185-206, 1990.

[25] Altenmueller, J., Mapping from EXPRESS to Physical File Structure. ISO TC184/SC4/WG1,Document N279, 1988

[20] Dunn, M., The STEP form features model, Proceedings of the CAM-I features symposium, P-90-PM-02, 1990.

[27] Chang, T.C., Expert process planning for manufacturing, Addison-Wesley, Reading, 1990.

[28] van Houten, F.J.A.M., van 't Erve, A.H., PART, a parallel approach to Computer Aided Process Planning, Proceedings of CAPE 4 Edinburgh, 1988.

[29] Chang, T.C., TIPPS - a Totally Integrated Process Planning System, Phd thesis, Virginia Polytechnic Institute, 1982.

[30] Hummel, K.E., Brooks, S.L., Symbolic representation of manufacturing features for an automated process planning system, ASME winter annual meeting, Anaheim, 1986

[31] van Houten, F.J.A.M., PART, a computer aided process planning system, Phd Thesis, University of Twente, 1992

[32] Iwata, K., Kakino, Y., Oba, F., Sugimura, N., Development of non-part family type computer aided production planning system CIMS/PRO, Advanced manufacturing technology, P. Blake, Ed., North Holland, 1980.

[33] Kakino, Y., Ohba, F., Moriwaki, T., Iwata, K., A new method of parts description for computer aided process planning, in Advances in computer aided manufacturing, McPherson, Ed., 197-213, North Holland, 1977.

[34] Jonkers, F.J.C.M., A software architecture for CAPP systems, PhD Thesis, University of Twente, 1992

[35] Detand, J., Kruth, J.P., Kempenaars, J., Pinte, J., Kreuzfeldt, J.: The Generation of Non-Linear Process Plans, 22nd CIRP International Seminar on Manufacturing Systems, Enschede, 1990.

[36] Tönshoff, H.K., Beckendorf, U., Anders, N.: A Process Description Concept for Process Planning, Scheduling and Job Control, Proceedings of the 22nd CIRP Intermational Seminar on Manufacturing Systems, Enschede, 1990.

[37] Iwata, K., Fukuda, Y., Sugimura, N., A proposal of knowledge base structure for integrated process planning system, Proceedings of the 22th CIRP international seminar on manufacturing systems, Enschede, 1990.

[38] Arentsen, A., The shop floor control concept and the production control system of ESPRIT project 809, Msc. thesis, University of Twente, 1990.

[39] Scholz-Reiter, B., CIM Interfaces, Concepts, standards and problems of interfaces in Computer Integrated Manufacturing, Chapman \& Hall, London, ISBN $0412451603,1992$. 